\title{
Brown-Sequard Syndrome
}

National Institute of Neurological Disorders and Stroke (NINDS)

\section{Source}

National Institute of Neurological Disorders and Stroke (NINDS). Brown-Sequard

Syndrome Information Page.

Brown-Sequard syndrome (BSS) is a rare neurological condition characterized by a lesion in the spinal cord which results in weakness or paralysis (hemiparaplegia) on one side of the body and a loss of sensation (hemianesthesia) on the opposite side. BSS may be caused by a spinal cord tumor, trauma (such as a puncture wound to the neck or back), ischemia (obstruction of a blood vessel), or infectious or inflammatory diseases such as tuberculosis, or multiple sclerosis. 